OPEN ACCESS

Edited by:

Chang-In Choi,

Pusan National University Hospital,

South Korea

Reviewed by:

Ravindra Deshpande,

Wake Forest School of Medicine,

United States

Vladimir Cuk,

Clinical Hospital Center Zvezdara,

Serbia

*Correspondence:

Qiangkang Lin

lin1428lin@163.com

Specialty section:

This article was submitted to

Surgical Oncology,

a section of the journal

Frontiers in Oncology

Received: 23 April 2021 Accepted: 28 June 2021

Published: 30 July 2021

Citation:

Yan W, Zhou H, Shi S, Lin J and Lin Q (2021) Association Between Chemotherapy and

Survival in $T 1$ Colon Cancer With Lymph Node Metastasis: A Propensity-Score Matched Analysis.

Front. Oncol. 11:699400.

doi: 10.3389/fonc.2021.699400

\section{Association Between Chemotherapy and Survival in T1 Colon Cancer With Lymph Node Metastasis: A Propensity-Score Matched Analysis}

\author{
Wangxin Yan, Huizhen Zhou, Si Shi, Jixu Lin and Qiangkang Lin* \\ Department of Colorectal and Anal Surgery, The Third Affiliated Hospital of Shanghai University, Wenzhou People's Hospital, \\ Wenzhou No. 3 Clinical Institute Affiliated to Wenzhou Medical University, Wenzhou, China
}

This study aimed to comprehensively examine the efficacy of chemotherapy in T1 colon cancer patients with lymph node metastasis.

Methods: The differences in categorical variables in colon cancer patients according to lymph node status were evaluated by Pearson's chi-square test. The Kaplan-Meier method was used to assess Cancer-specific survival (CSS) and overall survival (OS) with the log-rank test. Cox proportional hazards models were built, multivariate Cox regression analyses were performed with the hazard ratio (HR) and 95\% confidence interval $(\mathrm{Cl})$ to identify the potential independent prognostic factors. Propensity score matching was also undertaken to adjust for treatment bias due to measured confounders.

Results: Younger age (52.2\% VS. 43.0\% for $\leq 65$ years old, $\mathrm{p}<0.001)$, female gender (50.3\% VS. 46.8\% for female, p < 0.001), more lymph nodes harvested (68.1\% VS. 46.6\% for $\geq 12$ lymph nodes harvested, $p<0.001)$, Black race (13.6\% VS. 12.0\% for the Black race, $\mathrm{p}<0.001)$, and higher tumor grade $(14.2 \% \mathrm{VS} .5 .6 \%$ for grade III/IV, $\mathrm{p}<0.001$ ) were more prone to be diagnosed with lymph node involvement. The receipt of adjuvant chemotherapy following radical surgery significantly reduced the risk of colon cancerspecific mortality by $33.9 \%$ after propensity-score matching $(\mathrm{HR}=0.661,95 \% \mathrm{Cl}=0.476$ 0.917, $p=0.013)$.

Conclusions: Younger age, female gender, more lymph nodes harvested, Black race, and higher tumor grade were more prone to be diagnosed with lymph node involvement. The receipt of adjuvant chemotherapy following radical surgery also significantly decreased the risk of colon cancer-specific mortality by $33.9 \%$ in $\mathrm{T} 1$ colon cancer with lymph node involvement.

Keywords: chemotherapy, survival, T1, colon cancer, propensity score 


\section{INTRODUCTION}

Colon cancer is among the most common causes of cancer and cancer-related death (1). T1 colon cancer refers to carcinoma with invasion confined to the submucosa $(2,3)$. As reported, however, approximately $10 \%$ of $\mathrm{T} 1$ colon cancer patients experience lymph node metastases and require radical intestinal resection with lymph node dissection (4). Although the risk factors for lymph node metastasis in T1 colon cancer have been widely reported, differences in opinion do exist $(5,6)$.

The oncological outcomes of stage I colon cancer patients are generally excellent following curative surgery; however, the presence of lymph node metastasis represents a prognostic feature in poor prognosis. Among treatments, 5-FU-based chemotherapy has been demonstrated to have significant survival benefits for patients with lymph node metastasis (7-9). Despite this, some patients do not receive further chemotherapy following radical surgery (10). The available data of oncological outcomes in T1 node-positive $(\mathrm{N}+)$ patients is lacking. For example, the well-known MOSAIC study, assessing the impact of adjuvant chemotherapy for stage III colon cancer, did not include T1 disease (9).

$\mathrm{T} 1$ disease is relatively rare and represents a small proportion of cases of colon cancer. It has been reported that such patients account for 2 to 12 percent of all cases of colon cancer in colonoscopic studies (11-15). Therefore, a large population-based cohort is needed to evaluate the predictors for lymph node metastasis in $\mathrm{T} 1$ colon cancer following curative surgery. The present study aimed to comprehensively examine the efficacy of chemotherapy in T1 colon cancer patients with postoperative lymph node metastasis.

\section{MATERIAL AND METHODS}

Sponsored by the National Cancer Institute (NCI), the Surveillance, Epidemiology, and End Results (SEER) database included both the incidence, clinicopathological information, and survival characteristics of malignant tumors, and covered $28 \%$ of the US population from 18 established cancer registries across the USA. The SEER ${ }^{\star}$ Stat software, version 8.3.8 (Surveillance Research Program, National Cancer Institute) was utilized to acquire data for this population-based study from the SEER database. Because data from the SEER database were anonymous and publicly available, ethical approval was waived and informed consent was unnecessary in this study.

The baseline covariates included the year of diagnosis, tumor location, age at diagnosis, the number of lymph nodes harvested, race, gender, grade, and chemotherapy based on the postcode of patients. As seen in Figure S1, we identified patients diagnosed with colon cancer between 2004 and 2015. Patients who met the following criteria were excluded: (1) patient race was unknown, (2) no positive histological confirmation, (3) non-adenocarcinoma histologies, (4) lack of active follow-up, and (5) without radical surgery. Finally, the targeted population was patients diagnosed with stage T1NanyM0 colon cancer, who were included in our analyses. Further analysis was conducted in stage $\mathrm{T} 1 \mathrm{~N}+\mathrm{M} 0$ colon cancer patients.

\section{Statistical Analysis}

Patients' demographic and clinical characteristics were included as follows: year of diagnosis (2004-2007, 2008-2011 and 20122015), tumor location [right-sided colon (from caecum to transverse colon) and left-sided colon (from splenic flexure to rectosigmoid junction)], age at diagnosis ( $\leq 65$ years old and $>65$ years old), the number of lymph nodes harvested ( $\leq 11$ and $\geq 12$ ), race (white, Black and other), gender (male and female), grade (I/II, III/IV and unknown), and chemotherapy (no chemotherapy and chemotherapy).

Cancer-specific survival (CSS) and overall survival (OS) served as the endpoints. The differences of the categorical variables in colon cancer patients according to the lymph node status were analyzed by Pearson's chi-square test. The KaplanMeier method was used to assess the survival with the log-rank test. Cox proportional hazards models were built and multivariate Cox regression analyses were performed with hazard ratio (HR) and $95 \%$ confidence interval (CI) to identify the potential independent prognostic factors from the variables examined, with $\mathrm{P}$ value less than 0.20 in univariate analyses.

Patient demographic and clinicopathological features were not balanced due to the inherent deficits of the retrospective cohort. Propensity score matching, a statistical normalization method for analyzing observational data by estimating the effects of a large number of factors that could affect treatment allocation, were then generated to balance covariates in different groups and reduce selection bias due to confounding variables (16). To provide a more robust assessment of survival outcomes, propensity score matching was performed between stage $\mathrm{T} 1 \mathrm{~N}+\mathrm{M} 0$ colon cancer patients with and without the receipt of chemotherapy using a 1:1 nearest neighbor matching algorithm. The following variables were used to calculate propensity to receive chemotherapy: year of diagnosis, tumor location, age at diagnosis, the number of lymph nodes retrieved, patient race, gender, and tumor grade. Statistically significant levels were two-tailed and set at a P value of less than 0.05. Statistical analyses were conducted using the IBM Statistical Package for the Social Sciences (SPSS) version 23.0 software package for Windows (SPSS, Inc., Chicago, IL, USA).

\section{RESULTS}

\section{Patient Characteristics}

In total, 36595 eligible colon cancer patients met the inclusion criteria, of which 33633 (91.9\%) patients were diagnosed with lymph node-negativity and 2962 (8.1\%) patients were diagnosed with lymph node positivity; 12428 (34.0\%) patients were diagnosed between 2004 to 2007, 12496 (34.1\%) patients were diagnosed between 2008 to 2011 and 11671 (31.9\%) patients were diagnosed between 2012 to 2015; 17379 (47.5\%) patients were right-sided colon cancer and 19216 (52.5\%) patients were left-sided colon cancer; 15999 (43.7\%) patients were less than 65 years old and 20596 (56.3\%) patients were over 65 years old; 18891 (51.6\%) patients had less than 12 lymph nodes retrieved and $17704(48.4 \%)$ patients had more than 12 lymph nodes retrieved; 29051 (79.4\%) patients were of white race, 4437 
(12.1\%) patients were of Black race and 3107 (8.5\%) patients were other races; 19371 (52.9\%) patients were male and 17224 (47.1\%) patients were female; 29071 (79.4\%) patients were diagnosed with grade I/II and $2320(6.3 \%)$ patients were diagnosed with grade III/IV; 2414 (6.6\%) patients received chemotherapy and 34181 (93.4\%) patients did not. The demographic and clinical characteristics among the whole cohort are shown in Table 1. Our study found that those who were younger in age $(52.2 \%$ VS. $43.0 \%$ for $\leq 65$ years old, $\mathrm{p}<$ $0.001)$, had female gender $(50.3 \%$ VS. $46.8 \%$ for female, $\mathrm{p}<$ 0.001 ), more lymph nodes harvested (68.1\% VS. $46.6 \%$ for $\geq 12$ lymph nodes harvested, $\mathrm{p}<0.001)$, were of Black race $(13.6 \% \mathrm{VS}$. $12.0 \%$ for Black race, $\mathrm{p}<0.001)$, and who had a higher tumor grade $(14.2 \%$ VS. $5.6 \%$ for grade III/IV, p $<0.001)$ were more prone to be diagnosed with lymph node involvement.

\section{The Efficacy of Chemotherapy in $\mathrm{T} 1 \mathrm{~N}+$ Colon Cancer Patients Before Propensity Score Matching}

We then included $2962(8.1 \%) \mathrm{T} 1 \mathrm{~N}+$ colon cancer patients in further analyzes. As shown as Figure 1, the CSS curves of T1N+ colon cancer patients with and without the receipt of chemotherapy were generated using the Kaplan-Meier method. The CSS of T1N+ colon cancer patients with the receipt of chemotherapy was significantly better than those without the receipt of chemotherapy (94.3\% VS. $89.3 \%$ for 5 -year CSS rate, $\mathrm{p}<0.001$ ).

In an unadjusted Cox proportional hazards analysis, the cancerspecific mortality risk in patients with the receipt of chemotherapy was reduced by $48.1 \%(\mathrm{HR}=0.519,95 \% \mathrm{CI}=0.397-0.678$, $\mathrm{p}<0.001)$. Only variables with a $\mathrm{P}$ value less than 0.20 in unadjusted Cox analyses were then entered into multivariate Cox analyses, including information on the year of diagnosis, tumor location, age at diagnosis, patient race, gender, tumor grade, and whether they received chemotherapy. The results of multivariate analyses also showed that the cancer-specific mortality risk in patients with the receipt of chemotherapy was independently decreased by $46.0 \%(\mathrm{HR}=0.540,95 \% \mathrm{CI}=0.409-0.712$, $\mathrm{p}<0.001$; Table 2).

\section{The Efficacy of Chemotherapy in $\mathrm{T} 1 \mathrm{~N}+$ Colon Cancer Patients After Propensity- Score Matching}

As shown in Table 3, the clinicopathologic characteristics of $\mathrm{T} 1 \mathrm{~N}+$ colon cancer patients were compared according to the receipt of chemotherapy before propensity-score matching. The year of diagnosis (35.2\% VS. 29.3\% for 2012-2015, p < 0.001), left-sided colon cancer (56.3\% VS. $48.4 \%$ for left-sided colon, $\mathrm{p}<$ $0.001)$, younger age $(62.5 \%$ VS. $34.8 \%$ for $\leq 65$ years old, $\mathrm{p}<$ 0.001 ), more lymph nodes harvested (71.5\% VS. $62.4 \%$ for $\geq 12$ lymph node harvested, $\mathrm{p}<0.001)$, and higher tumor grade (15.0\% VS. $13.0 \%$ for grade III/IV, p < 0.001) were more prone to be associated with receipt of adjuvant chemotherapy in $\mathrm{T} 1 \mathrm{~N}+$ colon cancer patients.

In evaluating the effect of chemotherapy on the survival of $\mathrm{T} 1 \mathrm{~N}+$ colon cancer patients, to avoid the bias introduced by the retrospective design, we balanced the above demographic and clinical characteristics mentioned with propensity score matching. After matching by the ratio of $1: 1$, a total of 890

TABLE 1 | Clinicopathologic characteristics of T1 colon cancer patients according to the lymph node status.

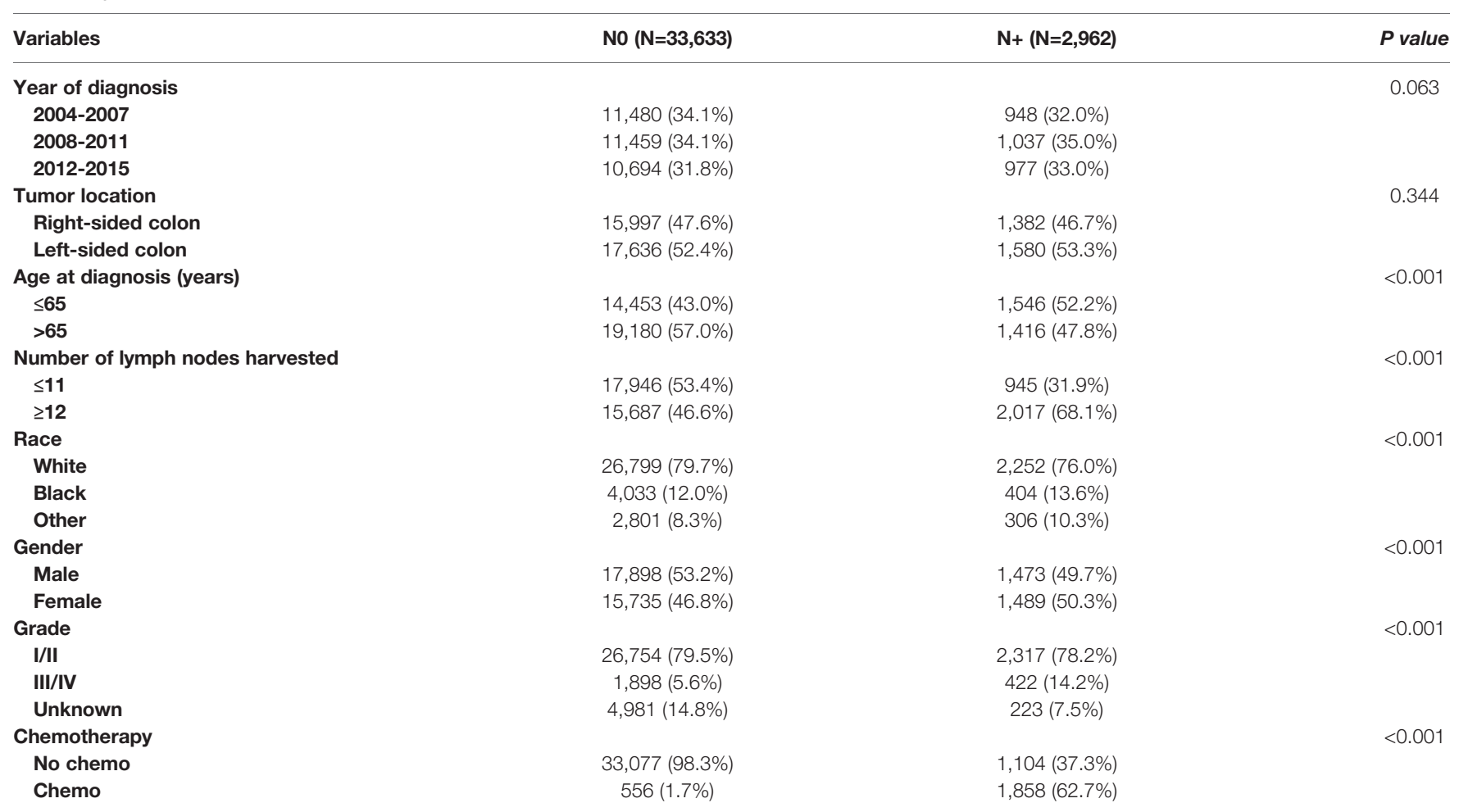




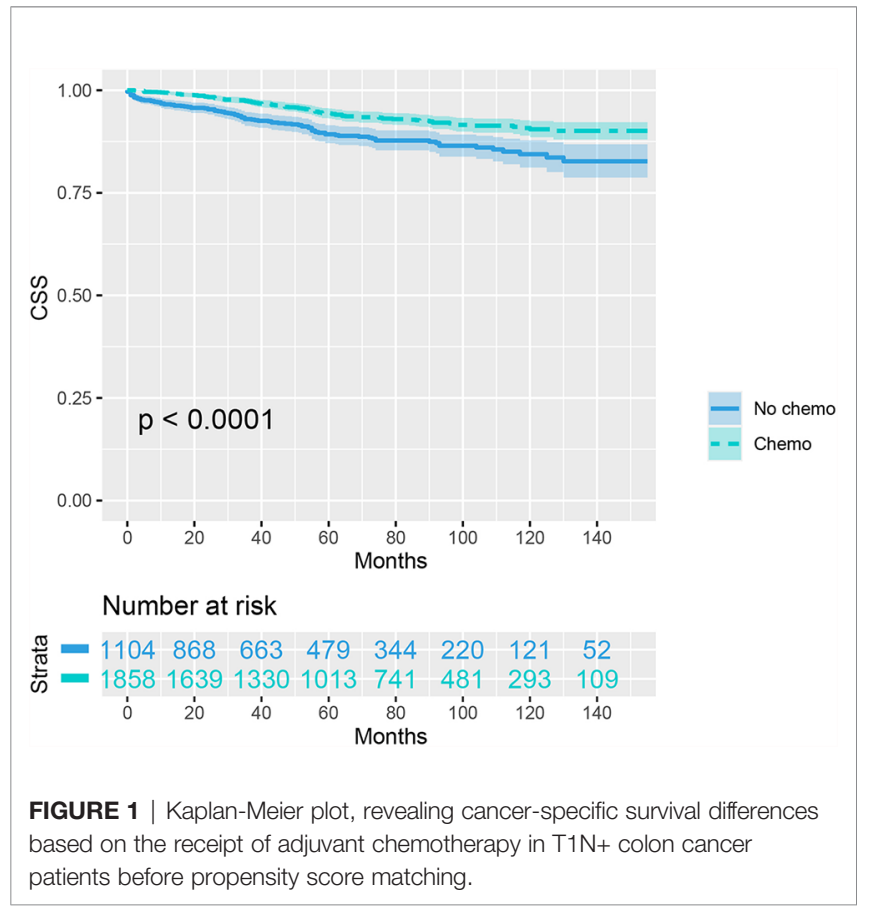

$\mathrm{T} 1 \mathrm{~N}+$ colon cancer patients with the receipt of chemotherapy were matched to $890 \mathrm{~T} 1 \mathrm{~N}+$ colon cancer patients without the receipt of chemotherapy. The distribution histograms before and after propensity-score matching are illustrated in Figure 2.
As indicated by Table 4, the clinicopathologic characteristics of $\mathrm{T} 1 \mathrm{~N}+$ colon cancer patients were compared according to the receipt of chemotherapy after propensity-score matching. Our study found that there was no difference between both groups with regards to year of diagnosis $(\mathrm{p}=1.000)$, tumor location $(\mathrm{p}=1.000)$, age at diagnosis $(\mathrm{p}=1.000)$, number of lymph nodes harvested $(p=1.000)$, patient race $(p=1.000)$, gender $(p=1.000)$ and tumor grade $(p=1.000)$. The receipt of adjuvant chemotherapy treatment following radical surgery did significantly decrease the risk of colon cancer-specific mortality by $33.9 \%$ after propensityscore matching $(\mathrm{HR}=0.661,95 \% \mathrm{CI}=0.476-0.917, \mathrm{p}=0.013)$. The Kaplan-Meier CSS curves of T1N+ colon cancer patients with and without the receipt of chemotherapy after propensity score matching are shown in Figure 3. The CSS of T1N+ colon cancer patients who received chemotherapy was significantly better than those who did not receive chemotherapy $(93.5 \%$ VS. $89.9 \%$ for 5 -year CSS rate, $\mathrm{p}=0.013$ ). Moreover, as seen in Figure 4, the $\mathrm{OS}$ of $\mathrm{T} 1 \mathrm{~N}+$ colon cancer patients who received chemotherapy was significantly better than those who did not (84.8\% VS. $66.3 \%$ for 5 -year OS rate, $\mathrm{p}<0.001)$.

\section{DISCUSSION}

In colon cancer, the presence of lymph node metastasis is a prognostic feature in poor prognosis configuration. In theory, lymph node metastasis should not occur when the tumor is confined to the mucosal layer because this layer is devoid of

TABLE 2 | Cox regression analysis of prognostic factors for cancer-specific survival in $\mathrm{T} 1 \mathrm{~N}+$ colon cancer.

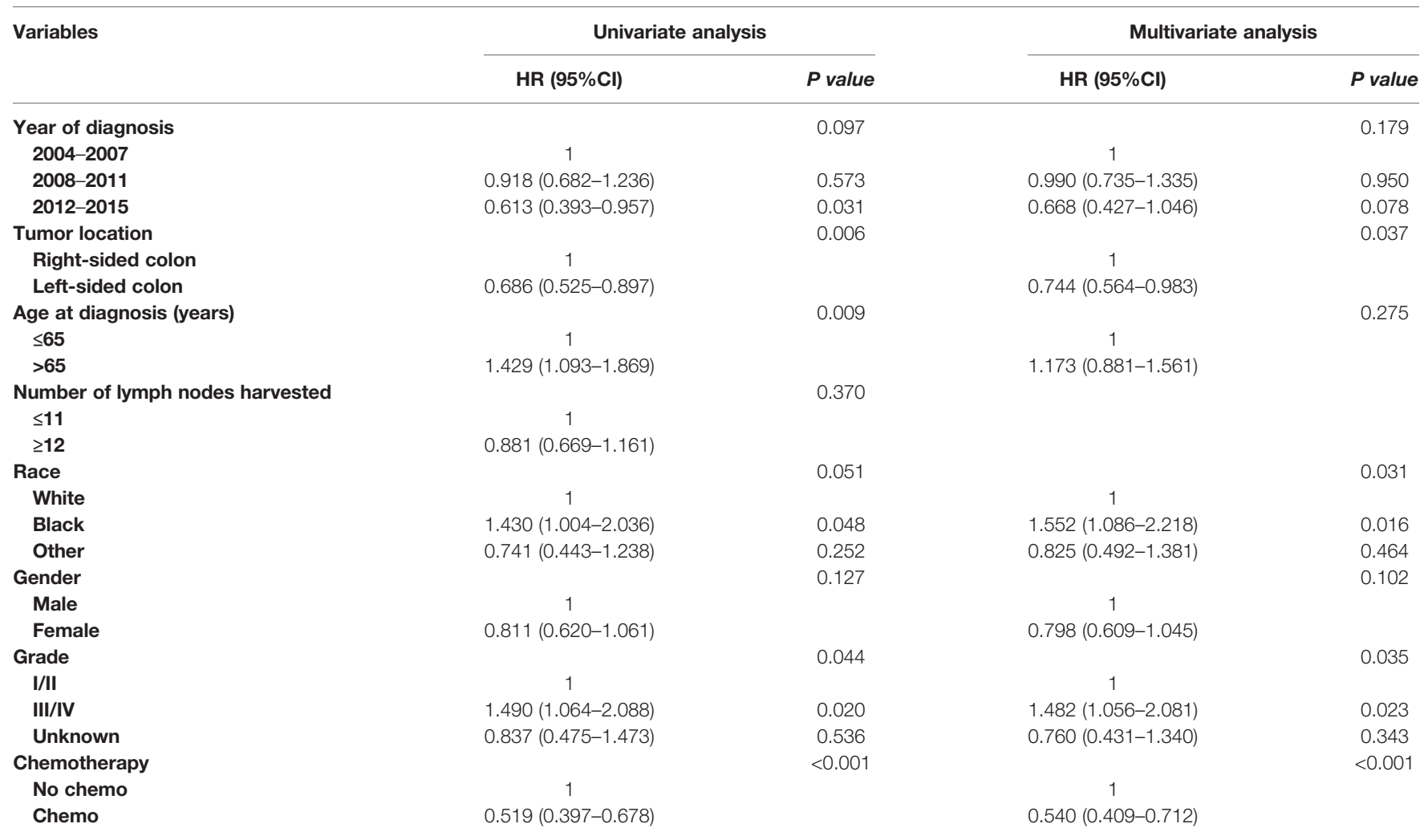


TABLE 3 | Clinicopathologic characteristics of T1N+ colon cancer patients according to the receipt of chemotherapy before propensity score matching.

\begin{tabular}{|c|c|c|c|}
\hline Variables & N0 chemo $(\mathrm{N}=1104)$ & Chemo ( $N=1858)$ & $P$ value \\
\hline Year of diagnosis & & & $<0.001$ \\
\hline 2004-2007 & $411(37.2 \%)$ & 537 (28.9\%) & \\
\hline 2008-2011 & $370(33.5 \%)$ & 667 (35.9\%) & \\
\hline 2012-2015 & $323(29.3 \%)$ & $654(35.2 \%)$ & \\
\hline Tumor location & & & $<0.001$ \\
\hline Right-sided colon & $570(51.6 \%)$ & 812 (43.7\%) & \\
\hline Left-sided colon & $534(48.4 \%)$ & $1046(56.3 \%)$ & \\
\hline Age at diagnosis (years) & & & $<0.001$ \\
\hline$\leq 65$ & $384(34.8 \%)$ & $1,162(62.5 \%)$ & \\
\hline$>65$ & $720(65.2 \%)$ & $696(37.5 \%)$ & \\
\hline Number of lymph nodes harvested & & & $<0.001$ \\
\hline$\leq 11$ & $415(37.6 \%)$ & $530(28.5 \%)$ & \\
\hline$\geq 12$ & 689 (62.4\%) & $1,328(71.5 \%)$ & \\
\hline Race & & & 0.090 \\
\hline White & $864(78.3 \%)$ & $1,388(74.7 \%)$ & \\
\hline Black & 137 (12.4\%) & 267 (14.4\%) & \\
\hline Other & 103 (9.3\%) & 203 (10.9\%) & \\
\hline Gender & & & 0.821 \\
\hline Male & 552 (50.0\%) & 921 (49.6\%) & \\
\hline Female & 552 (50.0\%) & 937 (50.4\%) & \\
\hline Grade & & & $<0.001$ \\
\hline I/II & 849 (76.9\%) & 1,468 (79.0\%) & \\
\hline III/IV & 143 (13.0\%) & 279 (15.0\%) & \\
\hline Unknown & $112(10.1 \%)$ & 111 (6.0\%) & \\
\hline
\end{tabular}

lymphatic vessels. T1 colon cancer, which refers to carcinoma with invasion confined to the submucosa, however, had an approximately $10 \%$ probability of experiencing lymph node metastases and therefore requires radical intestinal resection with lymph node dissection. Many studies have previously evaluated the risk factors for lymph node metastasis in T1 colon cancer, however, differences in opinion have always existed (3, 5, 17-21).

In our analyses, younger age, female gender, more lymph nodes harvested, Black race and higher tumor grade were more prone to be diagnosed with lymph node involvement. Two recent studies have reported that young age at diagnosis could be associated with an increased risk of lymph node involvement and more aggressive screening and postoperative treatments should be considered for young patients with T1 colon adenocarcinoma/ $(22,23)$ This phenomenon might be due to the potential genetic differences between young and elderly patients, as young patients are more likely to present with more aggressive features and adverse histological grades (2426) In line with the results of previous studies, we found that Black race had a risk factor of developing metastasis (23). The higher rate of lymph node metastasis in female colon cancer patients diagnosed with $\mathrm{T} 1$ disease might result from the sex

\section{Chemotherapy (before matching)}

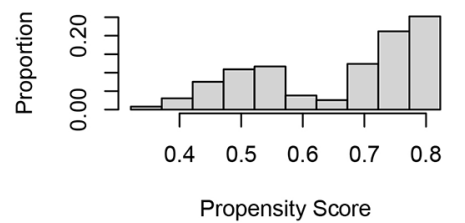

No chemotherapy (before matching)

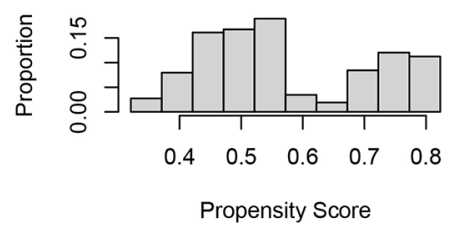

Chemotherapy (after matching)

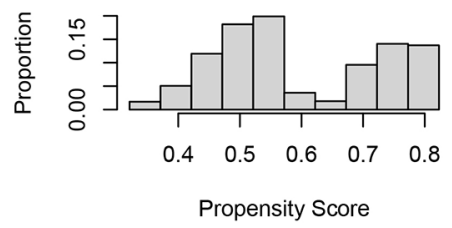

No chemotherapy (after matching)

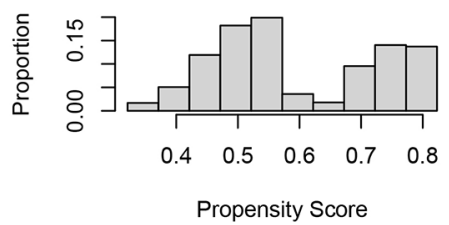

FIGURE 2 | Distribution histograms before and after propensity score matching (treated = no surgery; control = radical surgery). 
TABLE 4 | Clinicopathologic characteristics of T1N+ colon cancer patients according to the receipt of chemotherapy after propensity score matching.

\begin{tabular}{|c|c|c|c|}
\hline Variables & N0 chemo ( $N=890)$ & Chemo $(\mathrm{N}=890)$ & $P$ value \\
\hline Year of diagnosis & & & 1.000 \\
\hline 2004-2007 & 322 (36.2\%) & $322(36.2 \%)$ & \\
\hline 2008-2011 & 287 (32.2\%) & 287 (32.2\%) & \\
\hline 2012-2015 & $281(31.6 \%)$ & $281(31.6 \%)$ & \\
\hline Tumor location & & & 1.000 \\
\hline Right-sided colon & $440(49.4 \%)$ & $440(49.4 \%)$ & \\
\hline Left-sided colon & $450(50.6 \%)$ & $450(50.6 \%)$ & \\
\hline Age at diagnosis (years) & & & 1.000 \\
\hline$\leq 65$ & 356 (40.0\%) & $356(40.0 \%)$ & \\
\hline$>65$ & $534(60.0 \%)$ & $534(60.0 \%)$ & \\
\hline Number of lymph nodes harvested & & & 1.000 \\
\hline$\leq 11$ & $282(31.7 \%)$ & $282(31.7 \%)$ & \\
\hline$\geq 12$ & $608(68.3 \%)$ & $608(68.3 \%)$ & \\
\hline Race & & & 1.000 \\
\hline White & 715 (80.3\%) & $715(80.3 \%)$ & \\
\hline Black & 106 (11.9\%) & $106(11.9 \%)$ & \\
\hline Other & 69 (7.8\%) & $69(7.8 \%)$ & \\
\hline Gender & & & 1.000 \\
\hline Male & 445 (50.0\%) & 445 (50.0\%) & \\
\hline Female & 445 (50.0\%) & 445 (50.0\%) & \\
\hline Grade & & & 1.000 \\
\hline I/II & 717 (80.6\%) & 717 (80.6\%) & \\
\hline III/IV & 121 (13.6\%) & $121(13.6 \%)$ & \\
\hline Unknown & $52(5.8 \%)$ & $52(5.8 \%)$ & \\
\hline
\end{tabular}

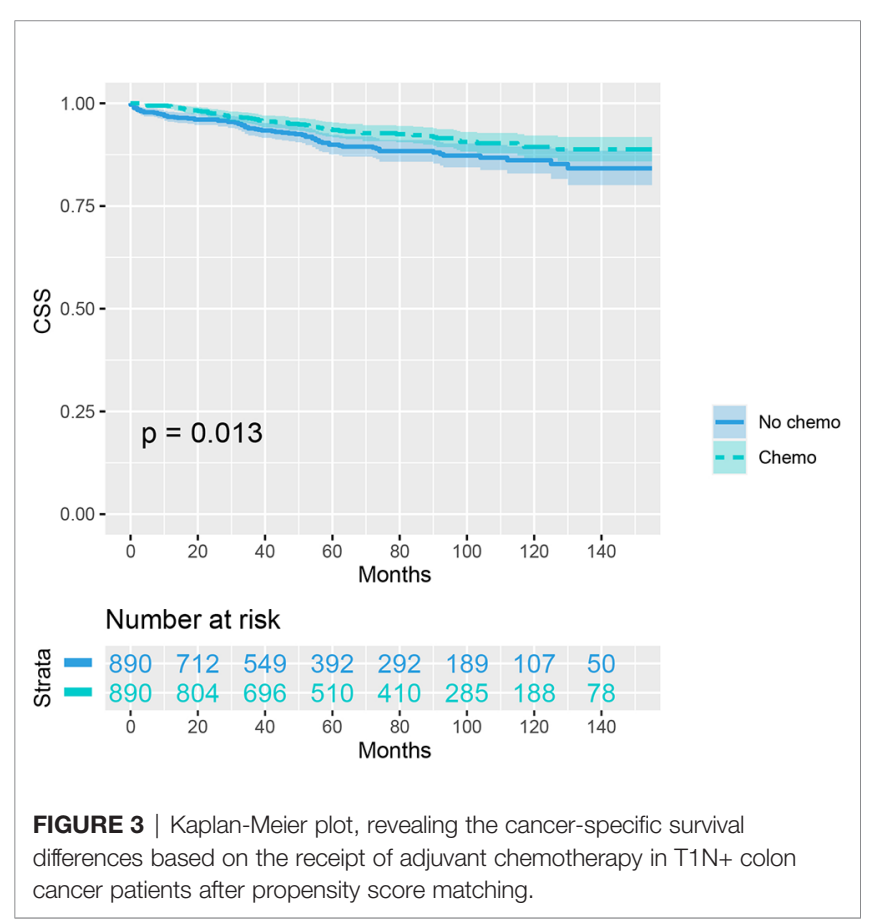

hormones between male and female patients $(27,28)$. It has also been observed that $\mathrm{T} 1$ carcinoma located in the left-sided colon shows higher rates of lymph node metastasis than right-sided colon, though it was not statistically significant in our study $(29,30)$.

According to current clinical guidelines, T1 colon cancer patients with lymph node metastasis should receive adjuvant chemotherapy following radical surgery. Moreover, 5-FU-based

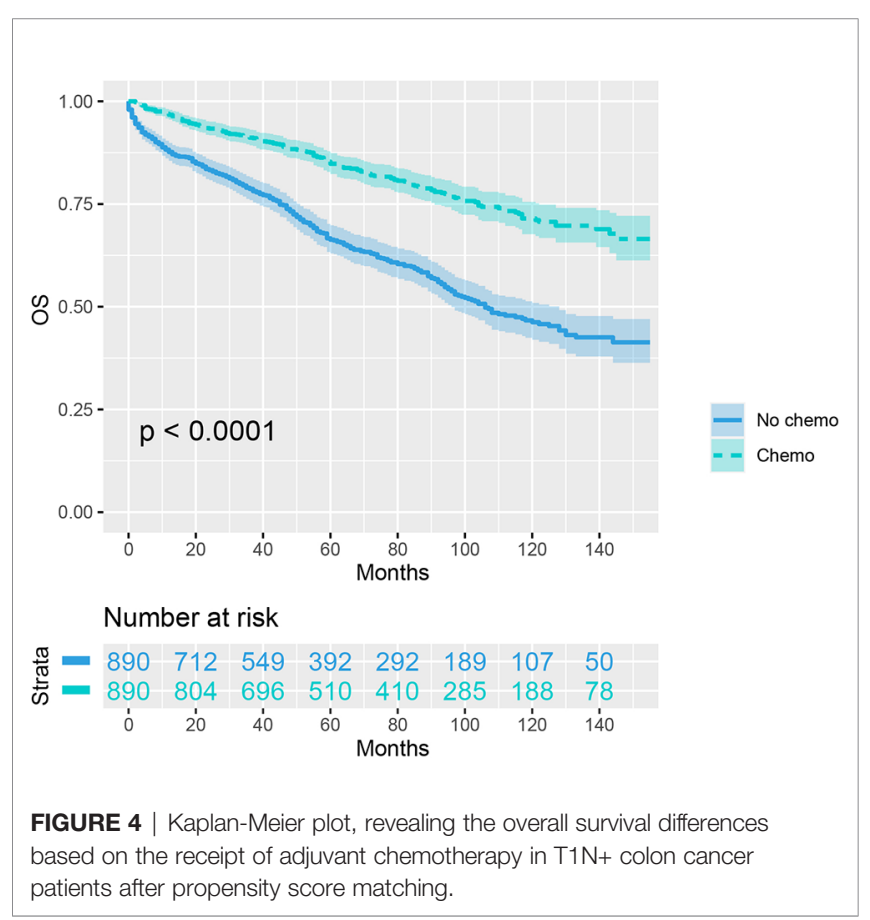

chemotherapy has been demonstrated to have significant survival benefits for patients with lymph node metastasis (3133). Early in 1990, Moertel and collaborators (8) demonstrated an improved prognosis of chemotherapy in colon carcinoma with lymph node metastasis following radical resection. Later in 2004, the famous MOSAIC study proposed that adding oxaliplatin to a regimen of fluorouracil and leucovorin provides improved efficacy in the adjuvant treatment of colon 
cancer (9). Cases of T1 disease are relatively few and account for a small proportion of colon cancer. It has been reported that such patients account for 2 to 12 percent of all colon cancer patients in colonoscopic studies (11-15). It is important to note that the above studies evaluating the efficacy of adjuvant chemotherapy for stage III colon cancer did not include T1 disease. However, T1 colon cancer patients with lymph node involvement following radical resection often did not receive further chemotherapy after surgery and the available data of oncological outcomes in T1 node-positive $(\mathrm{N}+)$ patients is lacking (10). In 2005, Wang et al. (34) evaluated the prognosis of T1 colorectal cancer in a small population $(n=159)$ and found that predictive factors for the risk of lymph node metastasis in T1 colorectal cancer after radical resection do not impact the long-term prognosis.

Despite findings such as these, studies on T1 colon cancer patients are mostly focused on the predictive factors for the risk of lymph node metastasis following radical resection $(5,6)$. T1 disease with lymph node involvement is much rarer than without lymph node metastasis, therefore, a large cancer database was required to examine the efficacy of chemotherapy in such patients. More importantly, our study has shown that adjuvant chemotherapy treatment could provide significantly better oncological outcomes in T1 colon cancer patients with lymph node involvement following radical surgery. In an unadjusted Cox proportional hazards analysis, the cancer-specific mortality risk in patients with the receipt of chemotherapy was reduced by $48.1 \%(\mathrm{p}<0.001)$. The results of multivariate analyses also showed that the cancer-specific mortality risk in patients who received chemotherapy was independently reduced by $46.0 \%$ $(\mathrm{p}<0.001)$. In addition, to reduce the bias introduced by the retrospective design, we balanced the demographic and clinical characteristics with propensity-score matching. The receipt of adjuvant chemotherapy treatment following radical surgery significantly reduced the risk of colon cancer-specific mortality by $33.9 \%$, even after propensity-score matching. Kaplan-Meier analysis also showed that the CSS of $\mathrm{T} 1 \mathrm{~N}+$ colon cancer patients with the receipt of chemotherapy was significantly better than those without the receipt of chemotherapy after propensity score matching, and the 5-year CSS rates were 93.5\%, and $89.9 \%$, respectively $(\mathrm{p}=0.013)$.

The major strengths of the current study are that it used a large cohort, and that we validated that younger age, female gender, more lymph nodes harvested, Black race, and higher tumor grade are more prone to be diagnosed with lymph node involvement. More importantly, by employing propensity score matching, the study provides a high level of evidence that the receipt of adjuvant chemotherapy following radical surgery significantly decreases

\section{REFERENCES}

1. Bray F, Ferlay J, Soerjomataram I, Siegel RL, Torre LA, Jemal A. Global Cancer Statistics 2018: GLOBOCAN Estimates of Incidence and Mortality Worldwide for 36 Cancers in 185 Countries. CA Cancer J Clin (2018) 68 (6):394-424. doi: 10.3322/caac. 21492

2. Okabe S, Shia J, Nash G, Wong WD, Guillem JG, Weiser MR, et al. Lymph Node Metastasis in T1 Adenocarcinoma of the Colon and Rectum. J Gastrointest Surg (2004) 8(8):1032-9. doi: 10.1016/j.gassur.2004.09.038 the risk of colon cancer specific mortality by $33.9 \%$ in $\mathrm{T} 1$ colon cancer with lymph node involvement.

Several limitations of the current study should also be noted. First, the information on postoperative complications, which could negatively affect the prognosis of colon cancer patients after radical resection was not included in the database, and could cause potential systematic bias. Second, the drawbacks introduced by the retrospective design could not be avoided, even though propensity-score matching was used. Finally, this database did not provide information on specific chemotherapy regimens, and further large-scale studies evaluating the effect of different chemotherapy regimens on survival in T1 colon cancer patients with lymph node metastasis are required.

\section{CONCLUSIONS}

Patients of younger age, female gender, more lymph nodes harvested, Black race, and higher tumor grade are more prone to be diagnosed with lymph node involvement. Using propensity-score matching, this study has provided important evidence that the receipt of adjuvant chemotherapy following radical surgery could significantly decrease the risk of colon cancer-specific mortality by $33.9 \%$ in $\mathrm{T} 1$ colon cancer with lymph node involvement.

\section{DATA AVAILABILITY STATEMENT}

Publicly available datasets were analyzed in this study. This data can be found here: https://seer.cancer.gov/.

\section{AUTHOR CONTRIBUTIONS}

WY and QL conceived the project and wrote the manuscript. SS and JL collected the data. WY, HZ, and SS undertook the analysis. All authors contributed to the article and approved the submitted version.

\section{SUPPLEMENTARY MATERIAL}

The Supplementary Material for this article can be found online at: https://www.frontiersin.org/articles/10.3389/fonc.2021.699400/ full\#supplementary-material

3. Nascimbeni R, Burgart LJ, Nivatvongs S, Larson DR. Risk of Lymph Node Metastasis in T1 Carcinoma of the Colon and Rectum. Dis Colon Rectum (2002) 45(2):200-6. doi: 10.1007/s10350-004-6147-7

4. Park YJ, Kim WH, Paeng SS, Park JG. Histoclinical Analysis of Early Colorectal Cancer. World J Surg (2000) 24(9):1029-35. doi: 10.1007/s002680010143

5. Hu DY, Cao B, Li SH, Li P, Zhang ST. Incidence, Risk Factors, and a Predictive Model for Lymph Node Metastasis of Submucosal (T1) Colon Cancer: A Population-Based Study. J Dig Dis (2019) 20(6):288-93. doi: $10.1111 / 1751-2980.12754$ 
6. Brunner W, Widmann B, Marti L, Tarantino I, Schmied BM, Warschkow R. Predictors for Regional Lymph Node Metastasis in T1 Rectal Cancer: A Population-Based SEER Analysis. Surg Endosc (2016) 30(10):4405-15. doi: 10.1007/s00464-016-4759-3

7. Zaniboni A. Adjuvant Chemotherapy in Colorectal Cancer With High-Dose Leucovorin and Fluorouracil: Impact on Disease-Free Survival and Overall Survival. J Clin Oncol (1997) 15(6):2432-41. doi: 10.1200/JCO.1997.15.6.2432

8. Moertel CG, Fleming TR, Macdonald JS, Haller DG, Laurie JA, Goodman PJ, et al. Levamisole and Fluorouracil for Adjuvant Therapy of Resected Colon Carcinoma. N Engl J Med (1990) 322(6):352-8. doi: 10.1056/ NEJM199002083220602

9. André T, Boni C, Mounedji-Boudiaf L, Navarro M, Tabernero J, Hickish T, et al. Oxaliplatin, Fluorouracil, and Leucovorin as Adjuvant Treatment for Colon Cancer. N Engl J Med (2004) 350(23):2343-51. doi: 10.1056/ NEJMoa032709

10. Steele SR, Park GE, Johnson EK, Martin MJ, Stojadinovic A, Maykel JA, et al. The Impact of Age on Colorectal Cancer Incidence, Treatment, and Outcomes in an Equal-Access Health Care System. Dis Colon Rectum (2014) 57(3):30310. doi: 10.1097/DCR.0b013e3182a586e7

11. Nusko G, Mansmann U, Partzsch U, Altendorf-Hofmann A, Groitl H, Wittekind $\mathrm{C}$, et al. Invasive Carcinoma in Colorectal Adenomas: Multivariate Analysis of Patient and Adenoma Characteristics. Endoscopy (1997) 29(7):626-31. doi: 10.1055/s-2007-1004268

12. Muto T, Sawada T, Sugihara K. Treatment of Carcinoma in Adenomas. World J Surg (1991) 15(1):35-40. doi: 10.1007/BF01658958

13. Nivatvongs S. Complications in Colonoscopic Polypectomy. An Experience With 1,555 Polypectomies. Dis Colon Rectum (1986) 29(12):825-30. doi: 10.1007/BF02555356

14. Hermanek P, Gall FP. Early (Microinvasive) Colorectal Carcinoma. Pathology, Diagnosis, Surgical Treatment. Int J Colorectal Dis (1986) 1 (2):79-84. doi: 10.1007/BF01648411

15. Shinya H, Wolff WI. Morphology, Anatomic Distribution and Cancer Potential of Colonic Polyps. Ann Surg (1979) 190(6):679-83. doi: 10.1097/ 00000658-197912000-00001

16. Lee YH, Lee YM, Sung TY, Yoon JH, Song DE, Kim TY, et al. Is Male Gender a Prognostic Factor for Papillary Thyroid Microcarcinoma? Ann Surg Oncol (2017) 24(7):1958-64. doi: 10.1245/s10434-017-5788-4

17. Huh JW, Kim HR, Kim YJ. Lymphovascular or Perineural Invasion may Predict Lymph Node Metastasis in Patients With T1 and T2 Colorectal Cancer. J Gastrointest Surg (2010) 14(7):1074-80. doi: 10.1007/s11605-010-1206-y

18. Rasheed S, Bowley DM, Aziz O, Tekkis PP, Sadat AE, Guenther T, et al. Can Depth of Tumour Invasion Predict Lymph Node Positivity in Patients Undergoing Resection for Early Rectal Cancer? A Comparative Study Between T1 and T2 Cancers. Colorectal Dis (2008) 10(3):231-8. doi: 10.1111/j.1463-1318.2007.01411.x

19. Bayar S, Saxena R, Emir B, Salem RR. Venous Invasion May Predict Lymph Node Metastasis in Early Rectal Cancer. Eur J Surg Oncol (2002) 28(4):413-7. doi: 10.1053/ejso.2002.1254

20. Goldstein NS, Hart J. Histologic Features Associated With Lymph Node Metastasis in Stage T1 and Superficial T2 Rectal Adenocarcinomas in Abdominoperineal Resection Specimens. Identifying a Subset of Patients for Whom Treatment With Adjuvant Therapy or Completion Abdominoperineal Resection Should Be Considered After Local Excision. Am J Clin Pathol (1999) 111(1):51-8. doi: 10.1093/ajcp/111.1.51

21. Xu X, Zhang C, Ni X, Wu J, Pan C, Wang S, et al. Population-Based Analysis on Predictors for Lymph Node Metastasis in T1 Colon Cancer. Surg Endosc (2020) 34(9):4030-40. doi: 10.1007/s00464-019-07192-0

22. Wang H, Lu H, Yang H, Zhang X, Thompson EW, Roberts MS, et al. Impact of Age on Risk of Lymph Node Positivity in Patients With Colon Cancer. J Cancer (2019) 10(9):2102-8. doi: 10.7150/jca.28377

23. Xie X, Yin J, Zhou Z, Dang C, Zhang H, Zhang Y. Young Age Increases the Risk for Lymph Node Metastasis in Patients With Early Colon Cancer. BMC Cancer (2019) 19(1):803. doi: 10.1186/s12885-019-5995-4
24. Chang DT, Pai RK, Rybicki LA, Dimaio MA, Limaye M, Jayachandran P, et al. Clinicopathologic and Molecular Features of Sporadic Early-Onset Colorectal Adenocarcinoma: An Adenocarcinoma With Frequent Signet Ring Cell Differentiation, Rectal and Sigmoid Involvement, and Adverse Morphologic Features. Mod Pathol (2012) 25(8):1128-39. doi: 10.1038/modpathol.2012.61

25. Barault L, Charon-Barra C, Jooste V, de la Vega MF, Martin L, Roignot P, et al. Hypermethylator Phenotype in Sporadic Colon Cancer: Study on a Population-Based Series of 582 Cases. Cancer Res (2008) 68(20):8541-6. doi: 10.1158/0008-5472.CAN-08-1171

26. Ogino S, Nosho K, Kirkner GJ, Kawasaki T, Meyerhardt JA, Loda M, et al. CpG Island Methylator Phenotype, Microsatellite Instability, BRAF Mutation and Clinical Outcome in Colon Cancer. Gut (2009) 58(1):90-6. doi: 10.1136/ gut.2008.155473

27. Bosetti C, Bravi F, Negri E, La Vecchia C. Oral Contraceptives and Colorectal Cancer Risk: A Systematic Review and Meta-Analysis. Hum Reprod Update (2009) 15(5):489-98. doi: 10.1093/humupd/dmp017

28. Grodstein F, Newcomb PA, Stampfer MJ. Postmenopausal Hormone Therapy and the Risk of Colorectal Cancer: A Review and Meta-Analysis. Am J Med (1999) 106(5):574-82. doi: 10.1016/S0002-9343(99)00063-7

29. Mochizuki K, Kudo SE, Ichimasa K, Kouyama Y, Matsudaira S, Takashina Y, et al. Left-Sided Location Is a Risk Factor for Lymph Node Metastasis of T1 Colorectal Cancer: A Single-Center Retrospective Study. Int J Colorectal Dis (2020) 35(10):1911-9. doi: 10.1007/s00384-020-03668-x

30. Aytac E, Gorgun E, Costedio MM, Stocchi L, Remzi FH, Kessler H. Impact of Tumor Location on Lymph Node Metastasis in T1 Colorectal Cancer. Langenbecks Arch Surg (2016) 401(5):627-32. doi: 10.1007/s00423-016$1452-\mathrm{x}$

31. Souglakos J, Boukovinas I, Kakolyris S, Xynogalos S, Ziras N, Athanasiadis A, et al. Three- Versus Six-Month Adjuvant FOLFOX or CAPOX for High-Risk Stage II and Stage III Colon Cancer Patients: The Efficacy Results of Hellenic Oncology Research Group (HORG) Participation to the International Duration Evaluation of Adjuvant Chemotherapy (IDEA) Project. Ann Oncol (2019) 30(8):1304-10. doi: 10.1093/annonc/mdz193

32. Liu Q, Luo D, Cai S, Li Q, Li X. Circulating Basophil Count as a Prognostic Marker of Tumor Aggressiveness and Survival Outcomes in Colorectal Cancer. Clin Trans Med (2020) 9(1):6. doi: 10.1186/s40169-019-0255-4

33. Chang SJ, Ge XS, Xu ZY, Qi XW, Chen XP. Lower Serum CA125 Level, Negative Vascular Invasion, and Wild BRAF Were Strongly Associated With Better 2-Year Disease-Free Survival in Patients With Stage III Colorectal Cancer Who Received Adjuvant Chemotherapy. Cancer Biomark (2018) 22 (1):161-8. doi: 10.3233/CBM-181179

34. Wang H-S, Liang W-Y, Lin T-C, Chen W-S, Jiang J-K, Yang S-H, et al. Curative Resection of T1 Colorectal Carcinoma: Risk of Lymph Node Metastasis and Long-Term Prognosis. Dis Colon Rectum (2005) 48(6):118292. doi: 10.1007/s10350-004-0935-y

Conflict of Interest: The authors declare that the research was conducted in the absence of any commercial or financial relationships that could be construed as a potential conflict of interest.

Publisher's Note: All claims expressed in this article are solely those of the authors and do not necessarily represent those of their affiliated organizations, or those of the publisher, the editors and the reviewers. Any product that may be evaluated in this article, or claim that may be made by its manufacturer, is not guaranteed or endorsed by the publisher.

Copyright (c) 2021 Yan, Zhou, Shi, Lin and Lin. This is an open-access article distributed under the terms of the Creative Commons Attribution License (CC BY). The use, distribution or reproduction in other forums is permitted, provided the original author(s) and the copyright owner(s) are credited and that the original publication in this journal is cited, in accordance with accepted academic practice. No use, distribution or reproduction is permitted which does not comply with these terms. 